\title{
Experiencia en reconstrucción mamaria inmediata con implante y matriz acelular de pericardio bovino tras mastectomía ahorradora de piel
}

\section{Experience in immediate breast reconstruction with implant and acellular hovine pericardium matrix after skin sparing mastectomy}

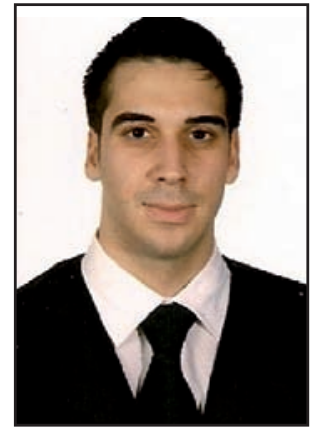

Aguilera Sáez, J.

\author{
Aguilera-Sáez, J.*, Bosacoma-Roura, P.**, Garrido-Ferrer, A.*, Guinot-Madridejos, A.**
}

Resumen

Presentamos nuestra experiencia en reconstrucción mamaria inmediata con implante y matriz acelular de pericardio bovino.

Realizamos un estudio que incluye todas las pacientes sometidas a reconstrucción mamaria inmediata con implante subpectoral y matriz acelular de pericardio bovino tras mastectomía ahorradora de piel, con o sin preservación del complejo areola-pezón, en la Unidad de Patología Mamaria del Hospital Universitario Vall d'Hebron de Barcelona (España) entre marzo de 2013 y diciembre de 2014.

En total se reconstruyeron 24 mamas, de las cuales se realizó mastectomía terapéutica en $17(70,8 \%)$ y mastectomía profiláctica en $7(29,2 \%)$. El volumen medio del implante colocado fue de 263,3 cc (rango de 80 a 445 cc). Siete mamas $(29,2 \%)$ fueron reconstruidas con expansor tisular y las otras $17(70,8 \%)$ con prótesis mamaria anatómica definitiva. La tasa global de complicaciones fue del 16,7\% (4 casos): 3 casos de seroma $(12,5 \%), 1$ caso de hematoma $(4,2 \%)$ y 1 de necrosis parcial del colgajo cutáneo $(4,2 \%)$ con extrusión del implante que requirió retirada del mismo. La tasa global de complicaciones en las mamas que recibieron radioterapia postoperatoria fue del 33,3\% (incluye el caso de fracaso reconstructivo).

En nuestra experiencia, la reconstrucción mamaria inmediata con implante y matriz acelular de pericardio bovino es una técnica segura y fiable, que permite obtener unos resultados óptimos con una tasa de complicaciones asumible y un menor coste económico.

Palabras clave Matriz acelular, Implantes mamarios, Reconstrucción mamaria inmediata.

Nivel de evidencia científica

\section{4}

The aim of this document is to share our experience in terms of immediate breast reconstruction using breast implants and acellular matrix made of bovine pericardium.

We carried out an study including all the patients treated at the Breast Pathology Unit of the Vall d' Hebron Hospital, Barcelona (Spain) who underwent an immediate breast reconstruction with a subpectoral breast implant and acellular matrix made of bovine pericardium after a skin sparing mastectomy with or without preservation of the nipple-areola complex, between March 2013 and December 2014.

A total of 24 breasts were reconstructed with this technique. Therapeutic mastectomy was made in 17 breasts $(70.8 \%)$ and 7 were prophylactic $(29.2 \%)$. The average volume of the implants was $263.3 \mathrm{cc}$ (range 80-445 cc ). Seven breasts $(29.2 \%)$ were reconstructed with a tissue expander and the other $17(70.8 \%)$ with a definitive anatomical breast implant. The overall complication rate was $16.7 \%$ (4 cases): 3 seromas cases $(12.5 \%), 1$ hematoma (4.2\%) and 1 partial skin flap necrosis $(4.2 \%)$ with extrusion of the implant which required removal. The overall complication rate in the breast that received postoperative radiation therapy was $33.3 \%$ (including the case of reconstructive failure).

In our experience, immediate breast reconstruction with breast implant and acellular matrix made of bovine pericardium is a safe and reliable technique, which allows the surgeon to obtain optimum results, with an acceptable rate of complications and a lower price.

\section{Key words Acellular matrix, Mammary implants,} Immediate breast reconstruction.

Level of evidence
4 


\section{Introducción}

La reconstrucción mamaria con prótesis o expansor tisular es el método más común de reconstrucción mamaria tras mastectomía. Supone por ejemplo el $37 \%$ de los casos de reconstrucción mamaria inmediata tras mastectomía en el Reino Unido (1). La reconstrucción con implantes ha evolucionado desde la colocación retromuscular completa a la colocación en un plano dual. Esto ha permitido una disminución del tiempo quirúrgico, mayor confort para las pacientes durante la expansión y menor morbilidad en la pared torácica. Sin embargo, esta técnica presenta menos control sobre el posicionamiento del surco submamario y la tasa de complicaciones asociadas puede acercarse al $40 \%$, incluyendo la extrusión y pérdida del implante, el desplazamiento o rotación del mismo, la contractura capsular y la formación de arrugas u ondulaciones en la piel $(2,3)$.

Las ventajas de la utilización de matriz dérmica acelular (MDA) en la reconstrucción mamaria con implante en posición subpectoral son: un mayor control del polo inferior con una mayor expansión del mismo y un mejor resultado estético con menos arrugas cutáneas. Sin embargo, múltiples estudios han comparado la reconstrucción mamaria con implante y MDA con la técnica tradicional y han observado una mayor incidencia de necrosis del colgajo de piel, infecciones, seromas y fracaso reconstructivo en el grupo de pacientes tratado con MDA (4).

El objetivo de este artículo es mostrar la experiencia del Servicio de Cirugía Plástica del Hospital Universitario Vall d’Hebron de Barcelona (España) con el uso de esta técnica quirúrgica, pero empleando una matriz acelular de pericardio bovino en lugar de una matriz dérmica acelular.

\section{Material y método}

Realizamos un estudio observacional, descriptivo, longitudinal y retrospectivo, que incluyó todas las pacientes sometidas a reconstrucción mamaria inmediata con implante más lámina acelular de pericardio bovino tras mastectomía ahorradora de piel o ahorradora de piel y preservadora del complejo areola-pezón (CAP), en la Unidad de Patología Mamaria del Hospital Universitario Vall d'Hebron de Barcelona (España), llevadas a cabo entre marzo de 2013 y diciembre de 2014.

Revisamos las historias clínicas de estas pacientes y analizamos los datos epidemiológicos, antecedentes patológicos, la presencia de radioterapia pre o postoperatoria, quimioterapia, la vía de abordaje de la mastectomía, el tipo y tamaño del implante colocado y las complicaciones postoperatorias: seroma, hematoma, dehiscencia de herida quirúrgica, necrosis cutánea, infección y fracaso reconstructivo por exposición del implante que obligara a la retirada del mismo (Tabla I).

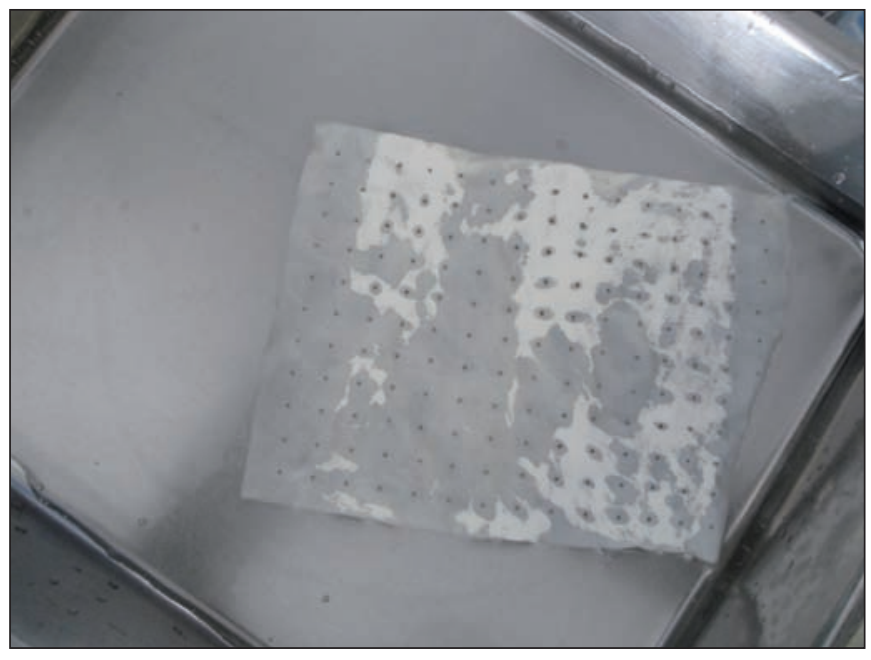

Fig. 1. Lámina de pericardio bovino sumergida en suero salino antes de su colocación.

La matriz de pericardio bovino Tutomesh ${ }^{\circledR}$ (Tutogen, Alemania) es una malla biológica perforada formada por fibras de colágeno tipo I entrelazadas tridimensionalmente, con un grosor de $0,5 \mathrm{~mm}$, deshidratada por solventes, libre de antígenos y de componentes celulares y esterilizada por rayos gamma (Fig. 1). Antes de su utilización se recomienda hidratar la malla sumergiéndola en suero salino estéril durante 4-5 minutos. Esta malla de pericardio bovino, de forma similar a las MDA, se reabsorbe gradualmente al mismo tiempo que se genera un tejido conectivo endógeno, actuando como una guía o un andamio para la formación de este tejido. En nuestro medio, presenta un coste económico inferior al de las MDA.

Estas matrices de pericardio bovino se han utilizado también durante los últimos años para otros procedimientos quirúrgicos, como por ejemplo la reconstrucción de la pared abdominal por hernias abdominales (5).

\section{Técnica quirúrgica}

Realizamos la técnica quirúrgica que explicamos a continuación tanto en las reconstrucciones mamarias inmediatas hechas directamente con prótesis definitiva, como en el primer tiempo quirúrgico de las realizadas con expansor tisular, variando únicamente el tipo de implante utilizado.

Tras la mastectomía conservadora de piel (con o sin preservación del CAP según el caso) llevada a cabo en nuestro centro por cirujanos generales o ginecólogos de la Unidad de Patología Mamaria, el equipo de Cirugía Plástica que forma parte de esta unidad multidisciplinar practica la reconstrucción mamaria inmediata. Inicialmente, seccionamos el origen del músculo pectoral mayor en la parrilla costal desde la posición de las 5 hasta las 8 horas imaginando un reloj analógico, y disecamos un bolsillo subpectoral poniendo especial interés en ligar las perforantes intercostales que lo irrigan (Fig. 2). El músculo aporta la cobertura para los dos tercios superiores del implante, seccionamos los orígenes esternales del pectoral mayor únicamente de forma parcial para conse- 


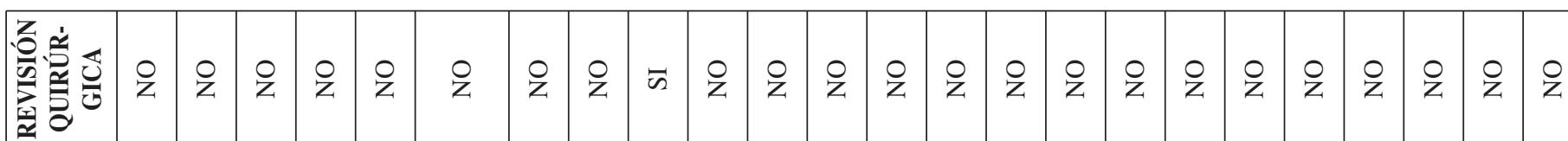

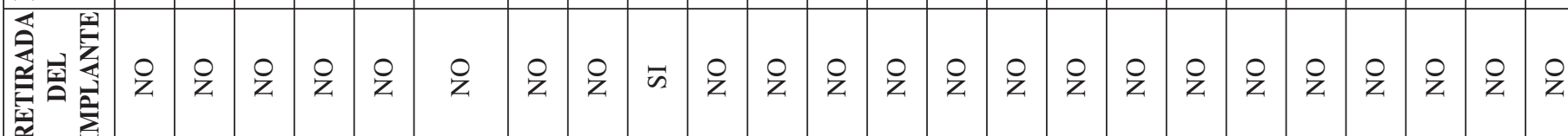

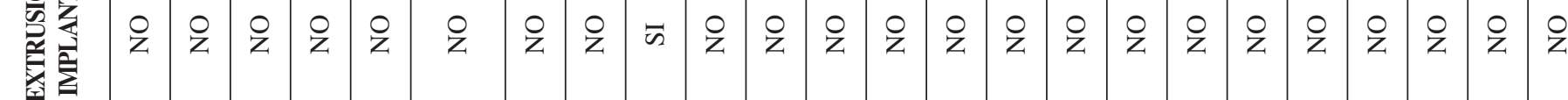

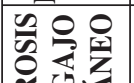

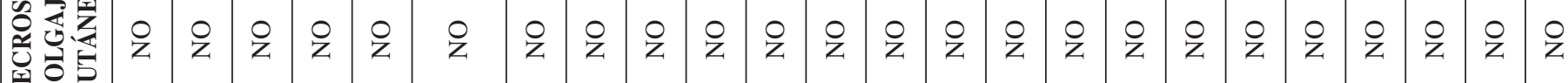

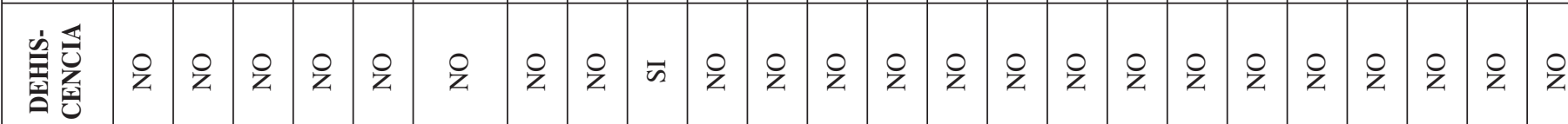

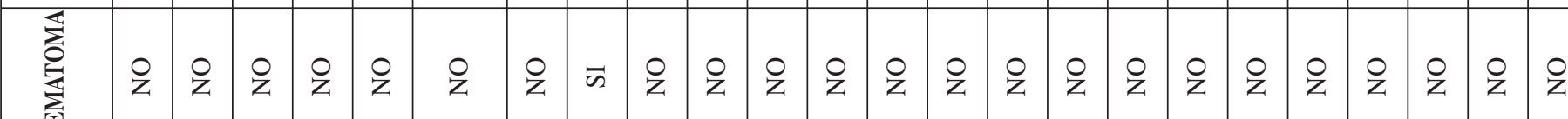

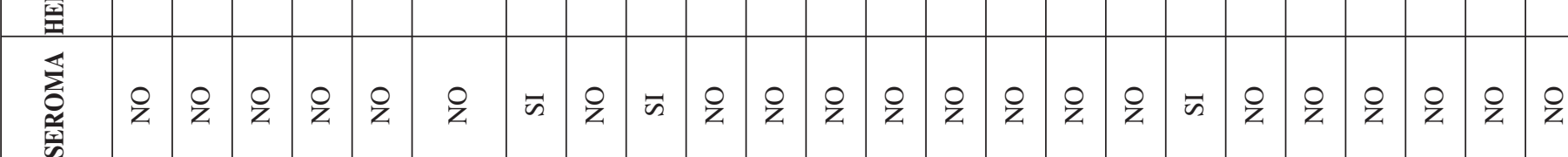

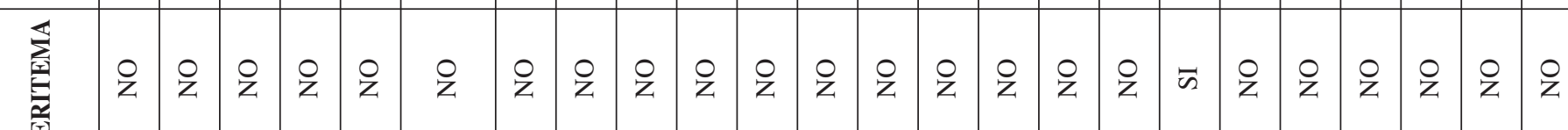



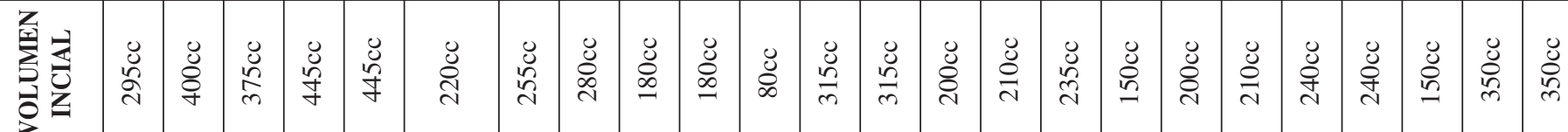

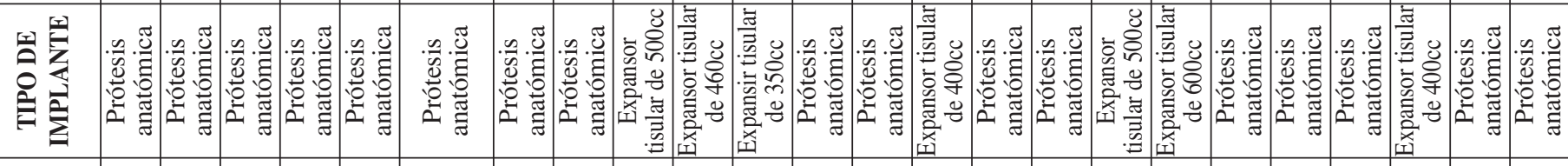

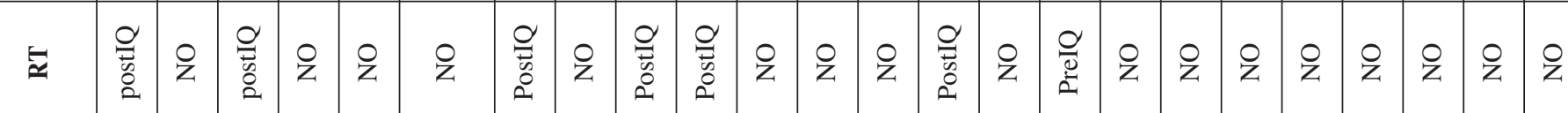

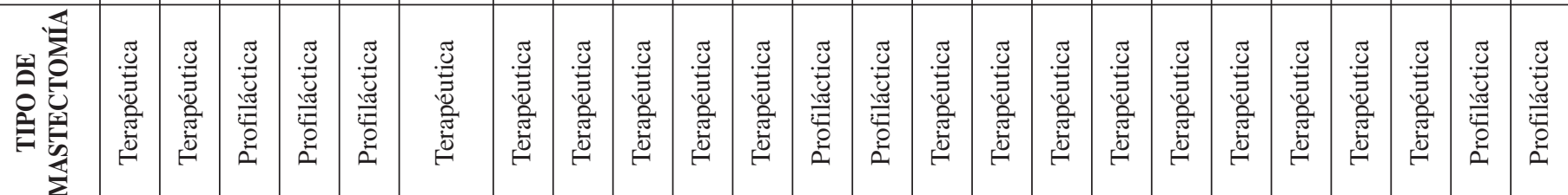

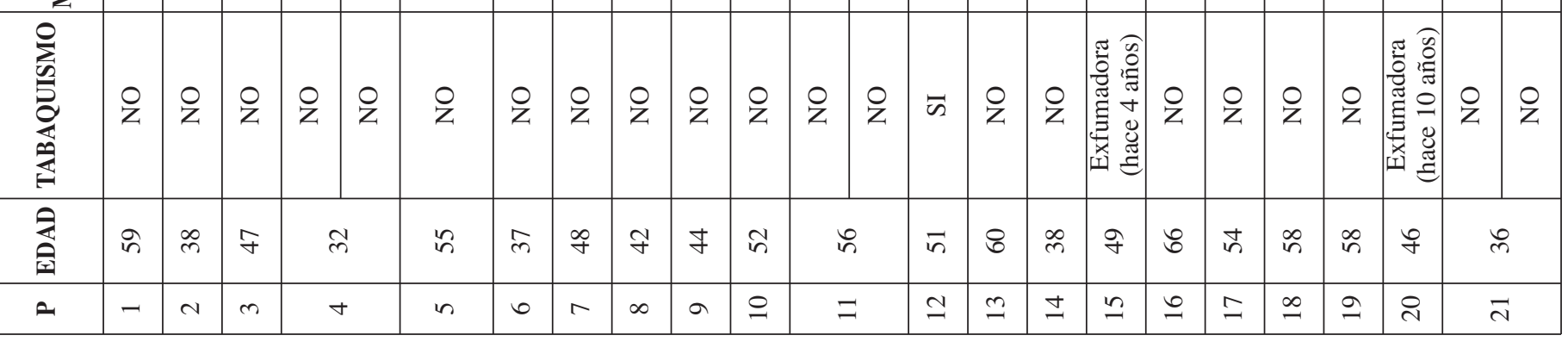




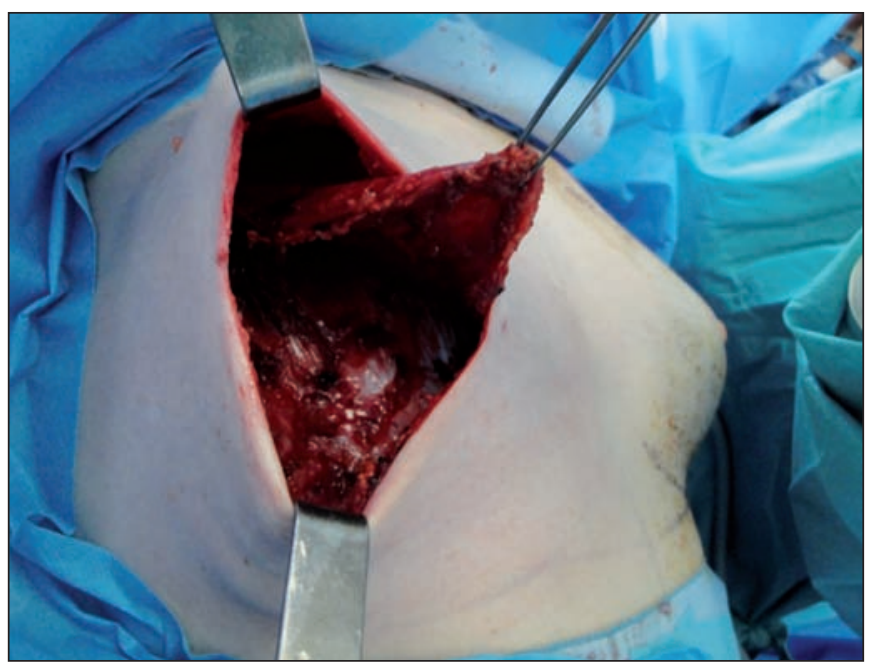

Fig. 2. Disección bolsillo subpectoral.

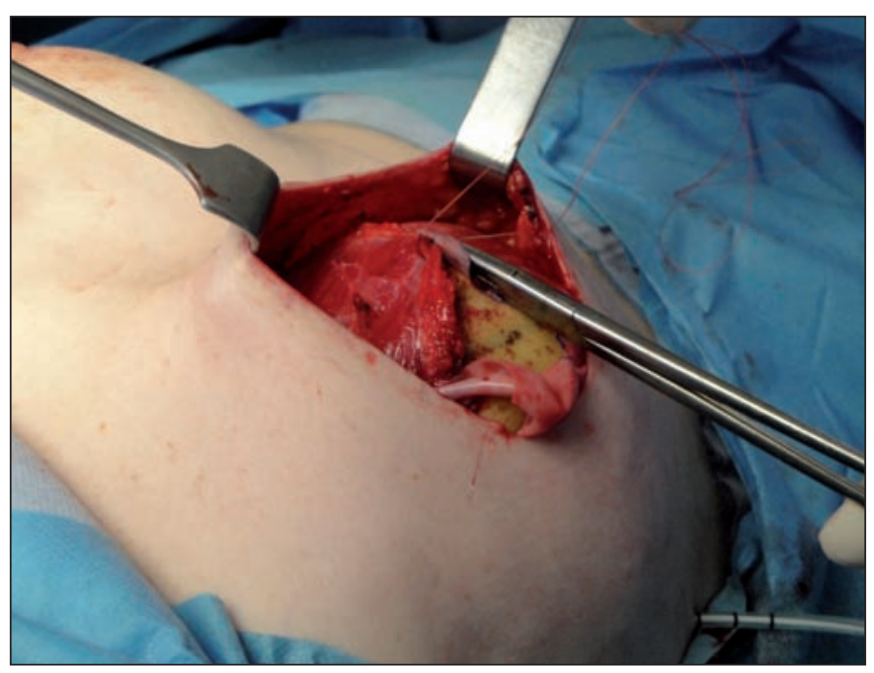

Fig. 4. Prótesis colocada bajo la matriz y sutura del borde distal del músculo pectoral al borde craneal de la matriz celular.

guir una proyección medial correcta, pero evitando la posibilidad de una sinmastia. Procedemos entonces a fijar la matriz de pericardio bovino a la fascia a nivel del surco submamario mediante puntos sueltos de Vycril 3.0 (Fig. 3). A continuación colocamos la prótesis o el expansor debajo del músculo y de la matriz, resecando los posibles excesos de la malla en los bordes, y procedemos a realizar una sutura continua con Vycril 3.0 del borde inferior del músculo pectoral mayor con el margen superior de la matriz (Fig. 4). Finalmente, recortamos los posibles excesos laterales de la matriz y ajustamos el margen lateral con puntos sueltos de Vycril 3.0 a la fascia del serrato, para que la malla nos haga también de sostén lateral y evitar así el desplazamiento lateral de la prótesis (Fig. 5). Colocamos un drenaje aspirativo subcutáneo y otro submuscular/submatriz. Retiramos el drenaje menos productivo a los 3 días (generalmente el subcutáneo) y el otro a los 5 días si el débito es inferior a 40 cc en 24 horas. Generalmente a la semana ya es poco productivo.

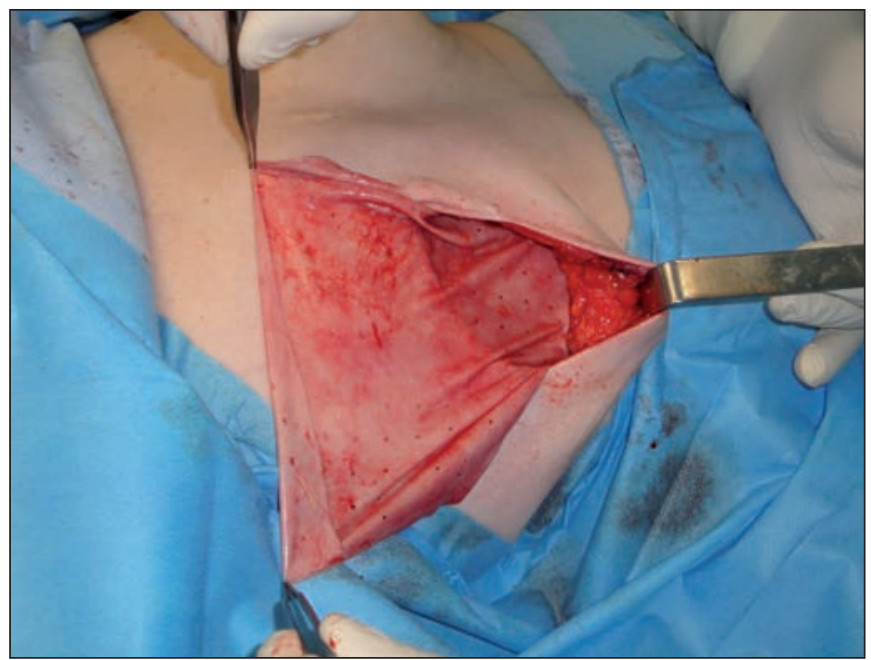

Fig. 3. Sutura de la matriz a la fascia a nivel del surco submamario.

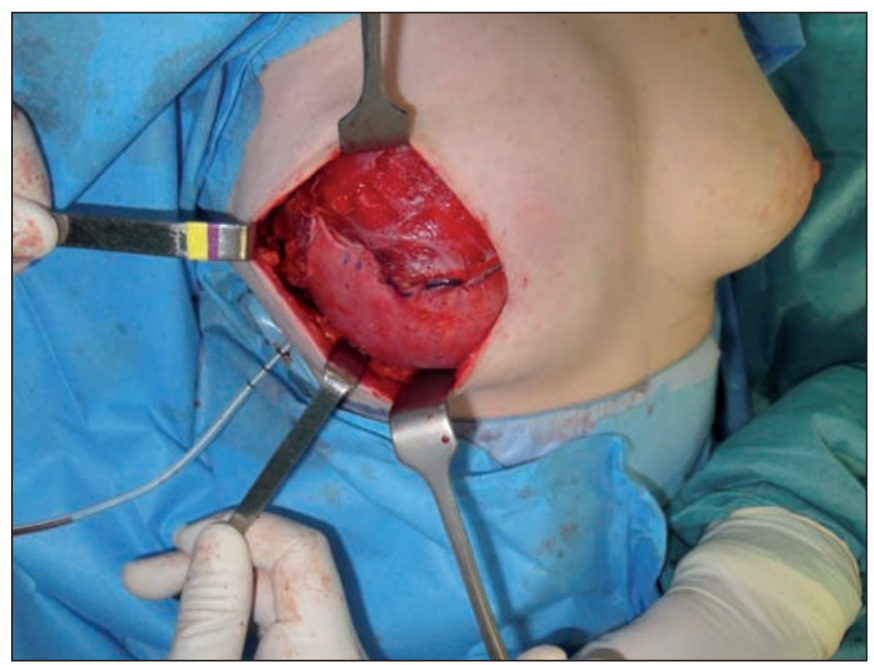

Fig. 5. Final del procedimiento con la matriz acelular correctamente colocada. Nótese como cubre por completo el tercio inferior del implante y el polo lateral.

\section{RESULTADOS}

Durante un periodo de 22 meses, entre marzo de 2013 y diciembre de 2014, 21 mujeres fueron sometidas en nuestra Unidad a mastectomía conservadora de piel con o sin preservación de CAP y reconstrucción mamaria inmediata con implante y matriz de pericardio bovino. Reconstruimos un total de 24 mamas, lo que representa 18 reconstrucciones unilaterales y 3 reconstrucciones bilaterales. La edad media de las pacientes fue de 48,4 años (rango de 32 a 66 años) y realizamos un seguimiento postoperatorio medio de 13,2 meses (rango de 6 a 25 meses). La mastectomía fue terapéutica en 17 mamas $(70,8 \%)$ y profiláctica en 7 mamas $(29,2 \%)$.

Solo 6 casos recibieron radioterapia postoperatoria y 1 caso había recibido radioterapia 25 años antes. En este último caso, la calidad de la piel era buena, sin signos evidentes de radiodermitis.

El volumen medio inicial de los implantes colocados fue de 263,3 cc (rango de 80 a $445 \mathrm{cc}$ ), teniendo en cuenta que 7 mamas $(29,2 \%)$ fueron reconstruidas con 

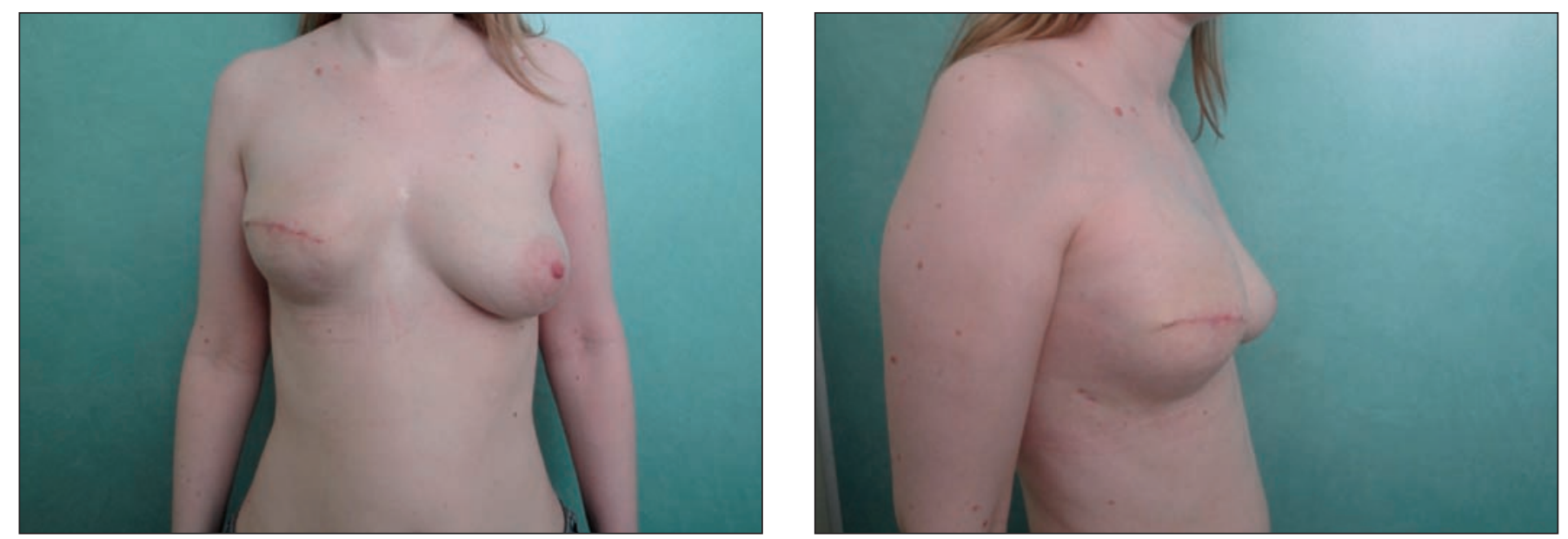

Fig. 6. Resultado de frente y perfil a las 6 semanas de postoperatorio tras mastectomía ahorradora de piel y reconstrucción mamaria inmediata con prótesis anatómica de $350 \mathrm{cc}$ y matriz dérmica de pericardio bovino.
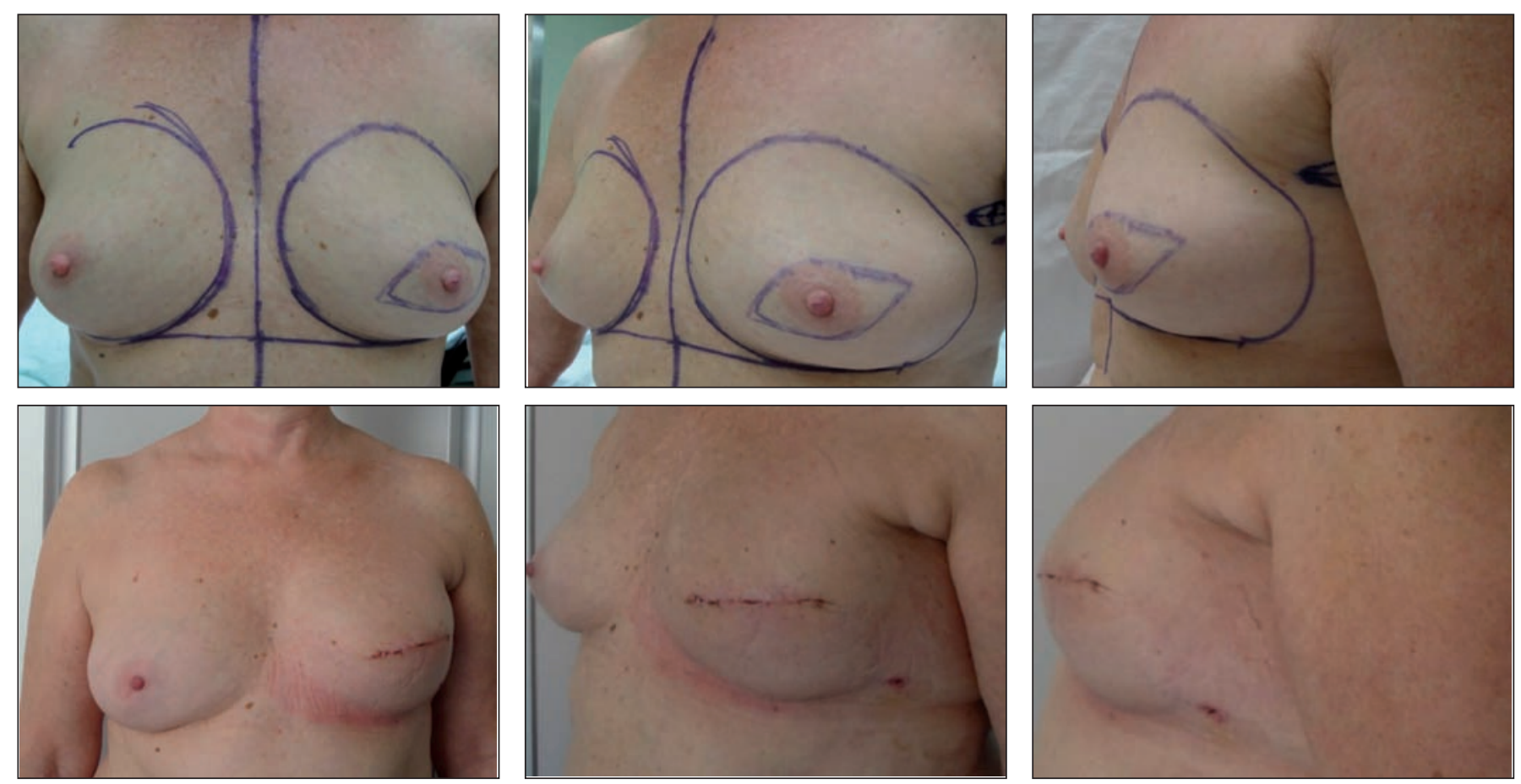

Fig. 7. Preoperatorio y postoperatorio inmediato en paciente sometida a reconstrucción mamaria inmediata con expansor tisular y matriz acelular de pericardio bovino. En la foto postoperatoria se ha realizado ya el recambio del expansor por prótesis anatómica definitiva.

expansor tisular y las otras 17 mamas $(70,8 \%)$ en un único tiempo quirúrgico con prótesis anatómica definitiva.

En cuanto a las complicaciones, de las 24 mamas reconstruidas aparecieron complicaciones en 4 (16,7\%), 2 en pacientes que recibieron radioterapia tras la intervención. Teniendo en cuenta que la radioterapia solo fue necesaria en 6 casos, supone una tasa global de complicaciones del 33,3\% en mamas sometidas a radioterapia postoperatoria y del $11,1 \%$ en mamas no sometidas a radioterapia, incluido en este grupo el caso de radioterapia preoperatoria puesto que habían pasado 25 años y la calidad de la piel era aparentemente correcta.

Recogimos 3 casos de seroma (12,5\%), de los cuales uno se resolvió satisfactoriamente tras drenaje con punción guiada por ecografía; otro, que se acompañó de ce- lulitis del colgajo cutáneo $(4,2 \%)$, se resolvió correctamente también tras drenaje con punción guiada por ecografía e inicio de terapia antibiótica; mientras que el tercero evolucionó hacia la necrosis parcial del colgajo cutáneo $(4,2 \%)$ con dehiscencia de la herida quirúrgica y extrusión del implante que requirió retirada del mismo $(4,2 \%)$ y está pendiente de reconstrucción diferida. Este caso corresponde a una de las mamas que recibió radioterapia tras la reconstrucción. Tuvimos también 1 caso de hematoma postquirúrgico $(4,2 \%)$ que se resolvió con evacuación en quirófano y revisión de la hemostasia.

Todos los casos realizados en 2 etapas quirúrgicas fueron completados satisfactoriamente tras la expansión completa, y en ningún caso tuvimos que realizar un colgajo de cobertura para rescate en un segundo tiempo quirúrgico (Fig. 6-7). 


\section{Discusión}

Las expectativas de las pacientes y los cirujanos en cuanto a la reconstrucción mamaria han ido incrementándose de forma paralela a la evolución de las técnicas reconstructivas. Una técnica de reconstrucción mamaria debe ser segura, fiable, predecible, provocar poca morbilidad y lograr un resultado estético excelente. En cuanto a la reconstrucción mamaria con implantes, su fiabilidad siempre ha sido un inconveniente.

Como hemos dicho anteriormente, la evolución hacia la posición retropectoral del implante que solo queda cubierto por el músculo en sus dos tercios superiores, ha permitido generar menos morbilidad en la pared torácica, mayor expansión del polo inferior y disminuir los tiempos quirúrgicos. Sin embargo, esta técnica presenta un menor control sobre el posicionamiento del surco submamario y su tasa de complicaciones asociadas puede ser alta, incluyendo la extrusión y pérdida del implante, el desplazamiento o rotación del mismo, la contractura capsular y la formación de arrugas u ondulaciones en la piel $(2,3)$. Hasta el $40 \%$ de estos casos complicados requerirá revisión quirúrgica (3).

La utilización de una matriz acelular presenta 3 ventajas teóricas evidentes. Por un lado, una matriz fijada al surco submamario permite un mayor control del posicionamiento del mismo. Por otro, la cobertura adicional que aporta al tercio inferior del implante disminuye el riesgo de extrusión y permite un mayor control del polo inferior, con una mayor expansión del mismo y un mejor resultado estético con menos arrugas cutáneas. Finalmente, la malla realiza una especie de efecto hamaca soportando el peso del implante y evitando el apoyo directo del mismo sobre la fina piel que lo recubre. A pesar de esto, en la literatura existen datos contradictorios al respecto.

Múltiples estudios han comparado la reconstrucción mamaria con implante asistida por MDA con la técnica tradicional y han observado una mayor incidencia de ciertas complicaciones. Por ejemplo, Ho y col., en su metanálisis publicado en 2012 (4), comparan la reconstrucción tradicional con implante y colgajos músculofasciales con la reconstrucción con implante y MDA incluyendo 4.876 mamas para concluir que la tasa de complicaciones en el grupo de reconstrucción mamaria asistida por MDA fue de un 10,9\% de necrosis del colgajo cutáneo, 6,9\% de seroma, 5,7\% de infección, 1,3\% de hematoma y $5,1 \%$ de fracaso reconstructivo. Los autores señalan por tanto que esta técnica presenta un riesgo mayor de seroma, infección y fallo reconstructivo. En cambio Spear y col. (6) refieren una tasa global de complicaciones del 12,1\% en las reconstrucciones mamarias asistidas por MDA y expansor tisular de una serie de 58 mamas $(3,4 \%$ de necrosis de colgajo cutáneo, $1,7 \%$ de seroma, $6,9 \%$ de infección y $1,7 \%$ de fracaso reconstructivo). Además no encontraron diferencias significativas entre la tasa de incidencia de las distintas complicaciones se hubiera usado o no MDA. Incluso Salzberg y col. (7) refieren una tasa global de complicaciones de solo el 3,9\% en una serie de 466 mamas con una tasa de necrosis cutánea y pérdida del implante mucho menor, inferior al 1,5\%, en comparación con lo reportado en la literatura para pérdida de implante en reconstrucciones convencionales (5-11\%).

Nuestra serie de reconstrucción mamaria inmediata con implante asociada a matriz acelular de pericardio bovino, pese a no presentar una tasa de complicaciones tan baja como la de Salzberg, sí presenta un porcentaje similar a la de Spear y muy por debajo del $40 \%$ comentado anteriormente. La tasa de necrosis cutánea y de pérdida del implante es algo menor a lo recogido en la literatura para reconstrucción convencional con implante $(4,2 \%$ frente a $5-11 \%)$. Creemos que en la formación de seromas, una de las principales complicaciones en todas las series publicadas de reconstrucción mamaria con prótesis y malla acelular, juega un papel fundamental la propia matriz acelular que podría actuar como cuerpo extraño hasta que se integra en el organismo, las reacciones que se producen hasta que esto sucede, y el doble espacio muerto secundario a la falta de coincidencia entre la envoltura cutánea y el volumen subyacente. Además, si el seroma es de tamaño suficiente, puede provocar un efecto mecánico de tensión sobre los colgajos cutáneos ya endebles de por sí en este tipo de cirugías, sobre todo tras mastectomía terapéutica. Este aumento de la tensión sobre el colgajo de mastectomía podría empeorar la vascularización de los colgajos cutáneos, favoreciendo la necrosis de los mismos y/o la dehiscencia de la herida quirúrgica, por lo que creemos que es fundamental conocer este hecho para diagnosticar cuanto antes un seroma y poder solventarlo. El que la malla empleada en nuestros casos sea perforada permite la comunicación entre el bolsillo subpectoral y la disección subcutánea, lo que creemos que dificulta la formación de seromas al facilitar su evacuación a través de los drenajes aspirativos colocados. Y el hecho de que sea una malla más fina que otras comercializadas $(0,5 \mathrm{~mm}$ de grosor frente a $1 \mathrm{~mm}$ o hasta $1,4 \mathrm{~mm}$ ) creemos que hace más rápido su proceso de integración.

Algunas pacientes sometidas a reconstrucción mamaria con MDA desarrollan un eritema postoperatorio suprayacente, fenómeno que se conoce como síndrome de la mama roja y que es fácilmente confundible con un proceso infeccioso. Este eritema presenta una respuesta escasa a la terapia antibiótica y en ocasiones responde al tratamiento con corticoides (8). Como etiología se ha propuesto una reacción de hipersensibilidad tardía (tipo IV o mediada por células) $(8,9)$. De las 24 mamas reconstruidas con matriz acelular de pericardio bovino en nuestro grupo de estudio no apreciamos este fenómeno en ningún caso. El tipo de material y su procesamiento (la forma de tratarlo para eliminar las células de la matriz extracelular, el crosslinking artificial y el proceso de es- 
terilización), pueden determinar una respuesta celular diferente en el huésped una vez implantada la matriz acelular $(10,11)$, aunque no hay estudios clínicos comparativos aleatorizados en este sentido. Existe la posibilidad de que, por el tipo de material o por el procesado que recibe esta matriz de pericardio bovino, sea menos inmunogénica que las matrices dérmicas.

En aquellas pacientes en las que se prevea radioterapia, debemos tener en cuenta el posible retraso del tratamiento adyuvante que puede producirse como resultado de las complicaciones. Además, la radioterapia postoperatoria se asocia a un incremento de las mismas de hasta 4 veces más de acuerdo con Salzberg y col. (7). También constatamos este hecho en nuestra serie, en la que los casos que recibieron radioterapia tras la reconstrucción presentaron una tasa de complicaciones del 33,3\%, incluido el caso de fracaso reconstructivo, mientras que los casos que no recibieron radioterapia adyuvante presentaron una tasa de complicaciones del 11,1\%. En los 6 casos que recibieron radioterapia postoperatoria optamos por esta técnica reconstructiva porque en el momento de la cirugía creímos que la posibilidad de que la radioterapia fuera necesaria era muy escasa. Además, se ha visto también que la radioterapia postoperatoria incrementa el riesgo de contractura capsular (7) tanto en casos de utilización de MDA como en los que no se usó. Pese a esto, se ha visto que la utilización de la MDA puede reducir la severidad de la contractura capsular (12).

Aunque somos conscientes de que el tamaño de nuestra muestra, con 24 reconstrucciones mamarias, es insuficiente para extraer conclusiones definitivas y con evidencia estadística, los datos recogidos nos orientan hacia un correcto control de las complicaciones postoperatorias con un buen resultado estético en las pacientes sometidas a reconstrucción mamaria inmediata postmastectomía ahorradora de piel empleando implante más matriz acelular de pericardio bovino, siempre y cuando realicemos una estricta selección de las pacientes. En nuestro centro hospitalario, toda paciente que se va a someter a una mastectomía ahorradora de piel seguida de una reconstrucción mamaria es discutida en sesión multidisciplinar. Si se decide realizar una reconstrucción mamaria inmediata con prótesis y matriz acelular, se le informa de la posibilidad de realizar una reconstrucción en dos tiempos o bien de optar por una reconstrucción diferida según la vascularización de los colgajos cutáneos que se observe durante el acto quirúrgico.

Salzberg y col. (7) proponen un algoritmo de decisión con el que estamos plenamente de acuerdo. En función de si se preserva el CAP o no y del tamaño de la mama, se decide qué abordaje de mastectomía realizar. Y lo más importante, según la calidad del colgajo de mastectomía observado intraoperatoriamente, se opta por realizar una reconstrucción mamaria diferida o bien por colocar un expansor tisular si ésta es precaria, o por colocar una prótesis definitiva si la calidad de los colgajos de mastectomía es buena (Esquema 1). A su vez, se informa a la paciente de que 
llevará drenajes aspirativos durante un mínimo de 5 días. Cuando se trata de pacientes fumadoras y exfumadoras se les informa del mayor riesgo de sufrimiento cutáneo, exposición del implante y fracaso de la reconstrucción mamaria en el caso de que opten por esta técnica reconstructiva. Únicamente se les someterá a la intervención si entienden y asumen ese mayor riesgo de complicaciones. También tenemos en cuenta a las pacientes exfumadoras porque suelen no serlo realmente o bien lo son desde un período relativamente corto de tiempo, y hemos observado, en un estudio aún pendiente de publicación, que en nuestro caso presentan una tasa de complicaciones similar a la de las pacientes fumadoras. En pacientes con mamas de gran tamaño solo realizamos esta técnica si aceptan una reducción mamaria contralateral y un resultado final con mamas de menor tamaño. En la literatura está descrito un aumento del riesgo de complicaciones en pacientes con un índice de masa corporal mayor de 30 (4), y una mayor tasa de infección en pacientes que presentaban un peso de la pieza de mastectomía superior a $600 \mathrm{~g}$ (13). Ambas condiciones suelen estar relacionadas, aunque no es totalmente necesario. Estas recomendaciones son parecidas a las publicadas en otras revisiones de la literatura $(7,14)$.

\section{Conclusiones}

En la evolución de la reconstrucción mamaria hacia la búsqueda de una técnica segura y fiable que pueda conseguir unos resultados estéticos óptimos, la reconstrucción mamaria inmediata con implantes asociada a matriz dérmica acelular parece haber encontrado su lugar en la actualidad. La aparición de nuevos materiales ya utilizados para otros tipos de cirugía, que realizan una función similar y resultan más económicos, puede jugar un papel fundamental en este campo. Sin embargo, a día de hoy, hacen falta más estudios que comparen estos materiales para ver si son similares en cuanto a resultados y fiabilidad.

En nuestra experiencia y tras el análisis retrospectivo de las 24 reconstrucciones mamarias inmediatas realizadas en nuestro Servicio empleando implante mamario y matriz acelular de pericardio bovino, consideramos que los resultados obtenidos orientan a que se trata de una técnica segura, predecible y fiable, con buenos resultados estéticos y con una tasa de complicaciones cuanto menos similar a las reportadas para reconstrucción mamaria inmediata asociada a matriz dérmica acelular, teniendo en cuenta que los datos existentes en la literatura son bastante contradictorios.

La selección estricta de las pacientes tributarias de este tipo de reconstrucción mamaria es, posiblemente, uno de los factores clave para el éxito del procedimiento. A saber: pacientes no fumadoras, no sometidas a radioterapia, con mamas de volumen medio o pequeño, e índice de masa corporal inferior a 30 y la aparente buena vascularización intraoperatoria de los colgajos cutáneos de la mastectomía.
Dirección del autor

Dr. Jorge Aguilera Sáez

Servicio de Cirugía Plástica y Quemados, Hospital Universitario Vall d'Hebron

Passeig de la Vall d'Hebron 119-129

08035 Barcelona, España.

Correo electrónico: jordiagui85@gmail.com

\section{Bibliografía}

1. Jeevan R., Cromwell D., Browne J., et al.: Third annual report of the national mastectomy and breast reconstruction audit 2010. Leeds: The NHS Information Centre; 2010.www.ic.nhs.uk/mbr.

2. Vardanian A.J., Clayton J.L., Roostaeian J., et al.: Comparison of implant based immediate breast reconstruction with and without acellular dermal matrix. Plast Reconstr Surg. 2011; 128:403e-410e.

3. Hvilsom G.B., Friis S., Frederiksen K., et al.: The clinical course of immediate breast implant reconstruction after breast cancer. Acta Oncologica. 2011; 50:1045-1052.

4. Ho G., Nguyen T.J., Shahabi A., et al.: A systematic review and metaanalysis of complications associated with acellular dermal matrix assisted breast reconstruction. Ann Plast Surg. 2012; 68:346-356.

5. Limpert J.N., Desai A.R., Kumpf A.L., Fallucco M.A., Aridge D.L.: Repair of abdominal wall defects with bovine pericardium. Ann J Surg. 2009; 198 (5):e60-e65.

6. Spear S.L., Parikh P.M., Reisin E., Menon N.G.: Acellular dermis-assisted breast reconstruction. Aesthetic Plast Surg. 2008; 32 (3):418-425.

7. Salzberg C.A., Ashikari A.Y., Koch R.M., ChabnerThompson E.: An 8-year experience of direct-toimplant immediate breast reconstruction using human acellular dermal matrix (AlloDerm). Plast Reconstr Surg. 2011; 127:514-524.

8. Ganske I., Hoyler M., Fox S.E., Morris D.J., Lin S.J., Slavin S.A.: Delayed hypersensitivity reaction to acellular dermal matrix in breast reconstruction: the red breast syndrome? Ann Plast Surg. 2014; 73 Suppl 2:S139-143.

9. Wu Q., Yao M., Pan Y., Qing C., Cao Q., Xu W.: Experimental study on the inflammatory and immune responses of xenogenic acellular dermal matrix transplantation combined with thin split-thickness skin autograft. Zhonghua Zheng Xing Wai Ke Za Zhi. 2002; 18(5):266-268.

10. López Cano M., Armengol Carrasco M., Quiles Pérez M.T., Arbós Vía M.A.: Implantes biológicos en la cirugía de las heridas de la pared abdominal. Cir esp. 2013; 91 (4):217-223

11. Martí Toro E. et al.: Un reto en reconstrucción mamaria. Cir.plást. iberolatinoam. 2012; 38 (1):1-7.

12. Israeli R., Feingold R.S.: Acellular dermal matrix in breast reconstruction in the setting of radiotherapy. Aesthetic Surg J. 2011; 31:51S-64S.

13. Lanier S.T., Wang E.D., Chen J.J., et al.: The effect of acellular dermal matrix use on complication rates in tissue expander/implant breast reconstruction. Ann Plast Surg. 2010; 64:674-678.

14. Martin L., O'Donoghue J.M., Horgan K., Thrush S., Johnson R., Gandhi A.: Acellular dermal matrix (ADM) assisted breast reconstruction procedures: Joint guidelines from the Association of Breast Surgery and the British Association of Plastic, Reconstructive and Aesthetic Surgeons. Eur J Surg Oncol. 2013; 39:425-429. 